Eurasian Journal of Physics and Functional Materials

$2021,5(2), 88-101$

\title{
Time-of-flight fission fragment spectrometer of the MAVR facility
}

\author{
D. Aznabayev*,1,2, S.M. Lukyanov ${ }^{1}$, Zh. Zeinulla ${ }^{1,2,3}$ \\ T. Issatayev ${ }^{1,2,4}$, V.A. Maslov ${ }^{1}$, K. Mendibayev ${ }^{1,2,5}$, \\ E.V. Melnik ${ }^{1}$, V.I. Smirnov ${ }^{1}$, S.S. Stukalov ${ }^{1}$, \\ A.V. Shakhov ${ }^{1,3}$ \\ ${ }^{1}$ Joint Institute for Nuclear Research, Dubna, Russia
}

${ }^{2}$ The Institute of Nuclear Physics, Ministry of Energy of the Republic of Kazakhstan, Almaty, Kazakhstan

${ }^{3}$ Dubna State University, Dubna, Russia

${ }^{4}$ L.N. Gumilyov Eurasian National University, Nur-Sultan, Kazakhstan

${ }^{5}$ Al-Farabi Kazakh National University, Almaty, Kazakhstan

E-mail: daur_is101@mail.ru

DOI: 10.32523/ejpfm.2021050202

Received: 05.04.2021 - after revision

A time-of-flight system was created to register the reaction products and perform correlation measurements on a high-resolution magnetic analyzer (MAVR). The facility is located on the channel of the extracted beam of the U-400 cyclotron at the Laboratory of Nuclear Reactions JINR. The results of measuring the parameters of the time-of-flight spectrometer obtained on the basis of measuring the spontaneous fission fragments velocities of ${ }^{252} \mathrm{Cf}$ are described. In addition, the results of the measurement of fission fragments in the ${ }^{48} \mathrm{Ca}+{ }^{238} \mathrm{U}$ reaction are presented. In this case, two time-of-flight arms were used, with the help of it is planned to carry out correlation measurements of induced fission fragments of a complex system. The created system also makes it possible to carry out correlation (three particles) measurements of fission fragments with particles registered in the focal plane of the MAVR magnetic spectrometer. Another purpose of the described system is to register elastically scattered ions, which is used as a monitoring system for the quality and composition of the beam hitting on the target of the MAVR facility.

Keywords: fission fragments, MCP detector, time-of-flight. 


\section{Introduction}

Multi-parameter facilities for correlation measurements are widely used in modern experiments for studying the properties of exotic nuclei and the mechanisms of nuclear reactions with heavy ions. For this purpose, the precision magnetic spectrometer MAVR [1-3] was created at the Flerov Laboratory of Nuclear Reactions. It is located on the stable and radioactive heavy ion beams channel of accelerator complex DRIBS in the FLNR, JINR [4,5]. The spectrometer allows to register the products of nuclear reactions at the front angles, as well as to identify products by their mass $A<60$ and atomic number $Z<40$ with absolute accuracy in the focal plane of the analyzer, which has a length of about 2 meters (more details on http:/ / flerovlab.jinr.ru/mavr/).

One of the physical problems which can be solved by the analyzer is the study of a new process discovered in the FLNR JINR. The process is the emission of charged particles at the maximum possible energy (near the kinematic limit for the two-body reaction mechanism) [6]. As a result of this process, a "cold" residual nucleus is formed that can divide after the departure of a fast particle. A time-of-flight spectrometer was created to carry out correlation measurements with fission products emitted at large angles which makes it possible to register products in a heavier range of $Z$ and $A$. The description and main characteristics of the created spectrometer are given below.

When registering and measuring the characteristics of reaction products on beams of radioactive nuclei, special requirements are imposed on the characteristics of this equipment [7-11], because of the complexity of low-intensity experiments $\left(10^{2}-10^{4} \mathrm{~s}^{-1}\right)$, (the large variety of reaction channels, the presence of neutron and gamma background, etc.). Under these conditions, one of the most effectively used methods for determining the energy of a particle is the timeof-flight method $[12,13]$, which consists in measuring the energy of a particle $E_{K}$ by the time-of-flight $t$ of the path length $l$ :

$$
E_{K}=E_{0}\left(\frac{1}{\sqrt{1-\beta^{2}}}-1\right)
$$

where $E_{0}$ is the rest energy of the ions, $\beta=V / c$ - relative ion velocity, $V=l / t$ - ion velocity [13].

The main element of the time-of-flight method is the starting detector, which must have a high time (subnanosecond) resolution, minimal braking losses when registering heavy reaction products, low sensitivity to the background of light particles and resistance to radiation damage. Such requirements are met by detectors based on microchannel plates (MCP). The main advantage of microchannel plates is a high resolution in terms of time of flight, a high coefficient of purification of reaction products from the initial beam, the intensity of which is higher by orders of magnitude, low sensitivity to gamma and neutron background and high registration efficiency. In addition, this type of detector introduces minimal distortion in the energy spread and angular scattering of the registered products.

The created time-of-flight system makes it possible to carry out correlation 
measurements of fission fragments with particles registered in the focal plane of the MAVR magnetic spectrometer. Another purpose of the described system is to register elastically scattered ions, which is used as a monitoring system for the quality and composition of the beam falling on the target of the MAVR facility. This allows us to measure the ion energy of the beam, the integral flux of particles that have passed through the target.

\section{Time-of-flight of fission fragments measuring method}

In studies of nuclear fission fragments, the time-of-flight method is usually used to measure the velocities of paired fragments. From the law of conservation of momentum [14] in the case of fission, it follows that the velocities of paired fragments are inversely proportional to their masses, which can be replaced with sufficient accuracy by their mass numbers. The velocity of the fragment $v$ is usually determined from measurements of the time of flight of the fragment $t$ of a given distance $l$. If the mass number $M_{f}$ of the fissionable nucleus is known, the mass number of the fragment is related to the measured values by the relations:

$$
M_{l, h}=M_{f} \frac{l_{h, l} / t_{h, l}}{\left(l_{l} / t_{l}+l_{h} / t_{h}\right)}
$$

where $M_{l}$ and $M_{h}$ are the masses of light and heavy fragments, $t_{l}$ and $t_{h}-$ the time-of-flight of light and heavy fragments, $l_{l}$ and $l_{h}$ distances, respectively. From expression (2), the total kinetic energy of the primary fragments can be calculated:

$$
T K E=M_{f} v_{1} \frac{v_{2}}{2}
$$

When measuring the time of flight of paired fragments, experimental data are obtained in the form of two-dimensional velocity spectra. The transition from the velocity to the mass and energy of the fragments is made unambiguously using the expressions $(2,3)$. It should be taken into account that this approach requires the use of the assumption of a two-body reaction mechanism and compliance with the condition that the mass of $M_{p}+M_{t}=M_{f 1}+M_{f 2}$, where $M_{p}, M_{t}$ and $M_{f i}$ are the masses of the ions of the incident beam, the target and the reaction products, respectively. In this paper, the choice of the method was made on the independent calculation of the mass $M$ of the registered products from the measured values of the velocity over the time of flight and energy according to the formula (1).

\section{Description of a time detector based on microchannel plates}

As mentioned above, detectors that give time marks about the beginning and end of the path length $l$ of the fragment passage, should produce pulses with 
a subnanosecond pulse form. Since the velocity of the fission fragments is on the order of $10^{9} \mathrm{~cm} / \mathrm{s}$, with a path length of $1 \mathrm{~m}$, it is necessary to have a time resolution of at least $10^{-9} \mathrm{~s}$ in order to obtain a mass resolution:

$$
\frac{\delta M}{M}=1 \%
$$

In addition, the starting detector should be thin enough so that when it passes, the fragments velocity changes with minimal changes.

The best time resolution is obtained with the help of detectors that register emission electrons, and therefore such detectors are most often used in experiments. Thin carbon or polymer foils are used to emit electrons. The electron yield is proportional to the specific ionization energy loss and for fission fragments reaches several hundred. It is sufficient for the formation of short subnanosecond pulses with an amplitude greater than the threshold of operation of discriminators with a tracking threshold $(>10 \mathrm{mV})$. This is achieved by using microchannel plates with a gain of $10^{3}$. To obtain a good time resolution, it is important to ensure the isochronous motion of electrons from the region of their formation to the detector. At first, this is done by increasing the velocity of the electrons. The average energy of the emission electrons is in the region of several electron volts. Before registration, the electrons are accelerated in an electric field to energy of several kilo electron volts. Secondly, the movement of electrons to the detector is carried out using a homogeneous magnetic field or an electrostatic mirror in such a way as to obtain the same length of the trajectory, i.e. isochronously. This method provides $100 \%$ efficiency of registration of fission fragments [15].

The use of the MCP imposes quite strict requirements on the vacuum system. For their normal operation, a pressure of at least $6.5 \cdot 10^{-4} \mathrm{~Pa}\left(5 \cdot 10^{-6}\right.$ torr $)$ is required. Compared to the PMT, the MCP has small dimensions, better time characteristics and significantly less sensitivity to magnetic fields.

So, as the starting detector in this work, an MCP detector similar to the one described in $[16,17]$ was chosen, which consists of a conversion foil, an accelerating grid, an electrostatic mirror, and an assembly of microchannel plates. The principle of operation of such a detector is the detected particle, when passing through the foil, knocks out electrons from it. The emission electrons are accelerated in the electric field between the foil and the accelerating grid to an energy of about $3 \mathrm{keV}$. In the gap between the two electrostatic mirrors, the accelerated electrons are isochronously rotated by 90 degrees and then fall on the assembly of the MCP. Since regardless of the passage of the particle through the input foil, the electrons have an isochronous trajectory due to the design of the electrostatic mirror. Therefore output time signal of the detector is positionally independent.

As an input foil, mylar films with a thickness of $150 \mu \mathrm{g} / \mathrm{cm}^{2}$ with a gold coating with a thickness of $30 \mu \mathrm{g} / \mathrm{cm}^{2}$ are used. The size of the input foil is $20 \times 30 \mathrm{~mm}$, the size of the MCP is $33 \mathrm{~mm}$ and the size of the working area of the MCP is $25 \mathrm{~mm}$. The detected particles pass through all the fields formed by the grids, practically without deviating from their original direction and without changing the initial velocity. Due to the use of relatively thin films as conversion 
foils, minimal distortions are introduced into the energy spread and angular scattering of the detected particles [18].

The microchannel plate is fixed in a fiberglass frame above the metallized anode. The output signal is taken from the anode. The voltage on the MCP detector is applied in the range from $-2.1 \mathrm{kV}$ to $-3 \mathrm{kV}$ to create an electrostatic field and eventually receive signals with the required amplitude. A schematic picture of the created MCP detector is shown in Figure 1.

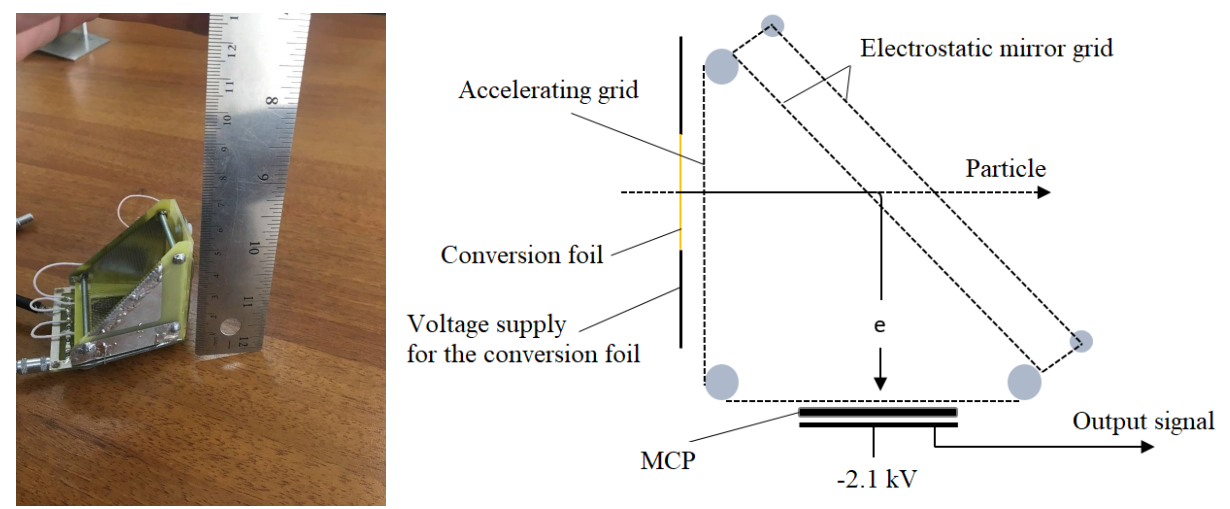

Figure 1. The scheme of MCP detector.

The electrostatic "mirror" is made of two planes of grids and CuBe wires with a thickness of $50 \mu \mathrm{m}$. The winding step is $1 \mathrm{~mm}$. The external grid of the electrostatic mirror is attached to the grooves of the support fiberglass plates [18].

\section{Measurement of the time-of-flight of alpha particles}

A radioactive source ${ }^{226} \mathrm{Ra}$ was selected for testing the efficiency and measuring the time-of-flight of particles using the MCP detector. The radioactive source ${ }^{226} \mathrm{Ra}$ emits alpha particles with known energy values $E_{\alpha_{1}}=7.6 \mathrm{MeV}, E_{\alpha_{2}}=6 \mathrm{MeV}$, $E_{\alpha_{3}}=5.4 \mathrm{MeV}, E_{\alpha_{4}}=4.8 \mathrm{MeV}$.

The test results are shown in Figure 2, which shows a two-dimensional matrix output of the registered alpha particles emitted by ${ }^{226} \mathrm{Ra}$, depending on their energy and time-of-flight:

The time-of-flight system used included a start and stop detector. The distance between the two detectors was set to $l=200 \mathrm{~mm}$. The work evaluated various ways of measuring the time-of-flight:

1. measurement of the time-of-flight of particles using two MCP detectors as the start and stop detectors;

2. measurement of the time of flight with one MCP detector as the start detector and Si detector as the stop detector. The comparative test results are shown in Figure 3.

Figure 3 shows the measured time-of-flight spectra of alpha particles from the ${ }^{226}$ Ra source obtained in these two TOF measurement methods: a) data from two MCP detectors, $b$ ) data from the MCP detector and Si detector for measuring the time-of-flight. Also Figure 3 shows that the time resolution for the system with MCP detectors (Figure 3a) is two times better than for the MCP-Si system 


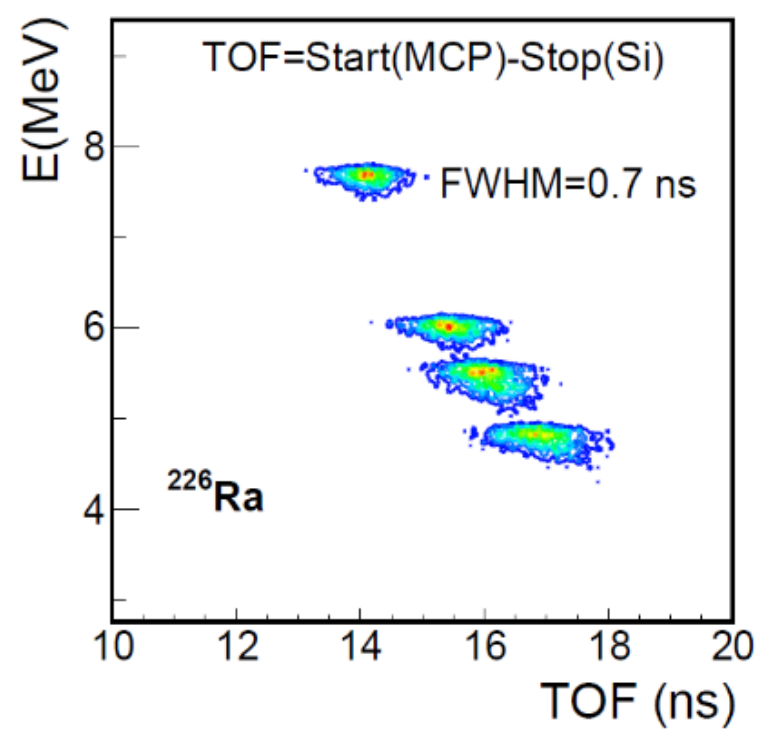

Figure 2. Two-dimensional matrix output of the registered alpha particles emitted by ${ }^{226} \mathrm{Ra}$, depending on their energy and time-of-flight.
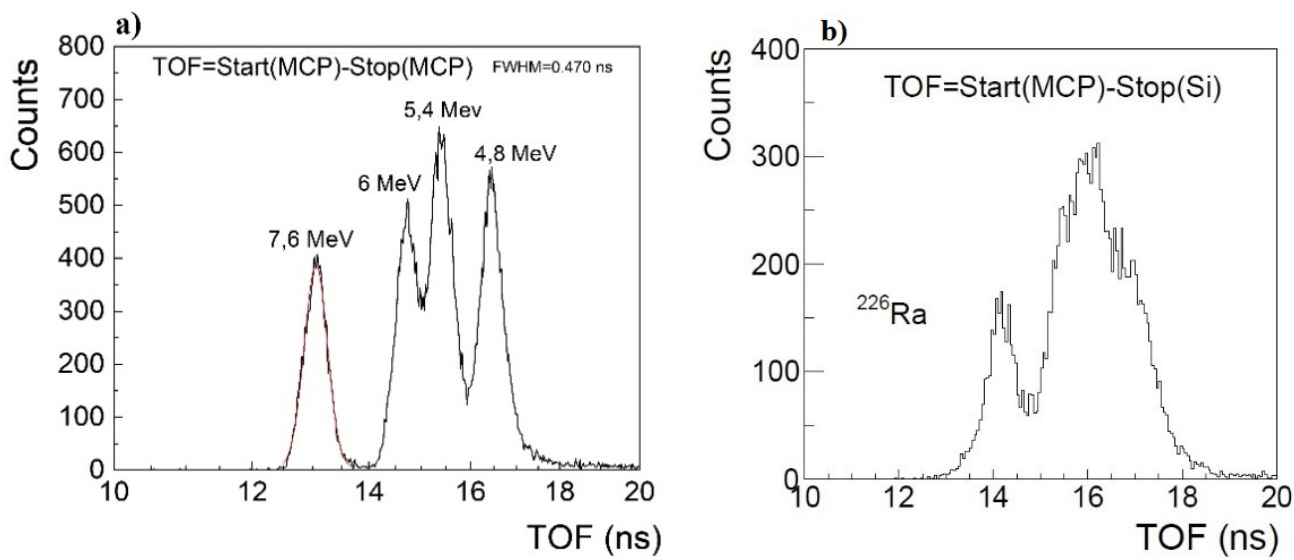

Figure 3. Time distribution of alpha particles emitted by ${ }^{226} \mathrm{Ra}$ : a) data obtained using two MCP detectors for the start and stop timestamps; b) data from the MCP detector and Si detector for measuring the time-of-flight.

(Figure $3 b$ ). In the first case, a temporary resolution of FWHM $=470 \mathrm{ps}$ was obtained. The use of a silicon semiconductor detector to obtain "stop" mark leads to a deterioration in the time resolution of almost 2 times (FWHM $=700$ ps). The deterioration of the time resolution is explained by the large pulse rise front (about hundreds of ns) of the semiconductor detector, as well as the size of the detector itself $\left(55 \times 55 \mathrm{~mm}^{2}\right)$. It is possible to improve this resolution by correcting the value of the path length when using strip detectors in the future.

\section{Registration of spontaneous fission fragments of ${ }^{252} \mathrm{Cf}$}

Spontaneous fission fragments of ${ }^{252} \mathrm{Cf}$ were also measured to test the time-offlight spectrometer and measure its characteristics. The main type of ${ }^{252} \mathrm{Cf}$ decay is $\alpha$-decay, and the contribution of competing spontaneous fission is quite high $3.08 \%$ (Figure 4).

Figure 5 shows a general view of the time-of-flight spectrometer used for 
measurements.

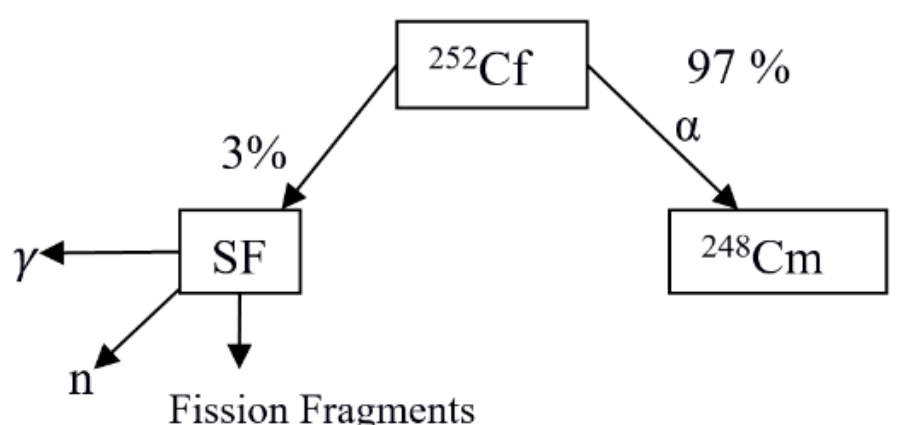

Figure 4. Decay scheme of ${ }^{252} \mathrm{Cf}$.

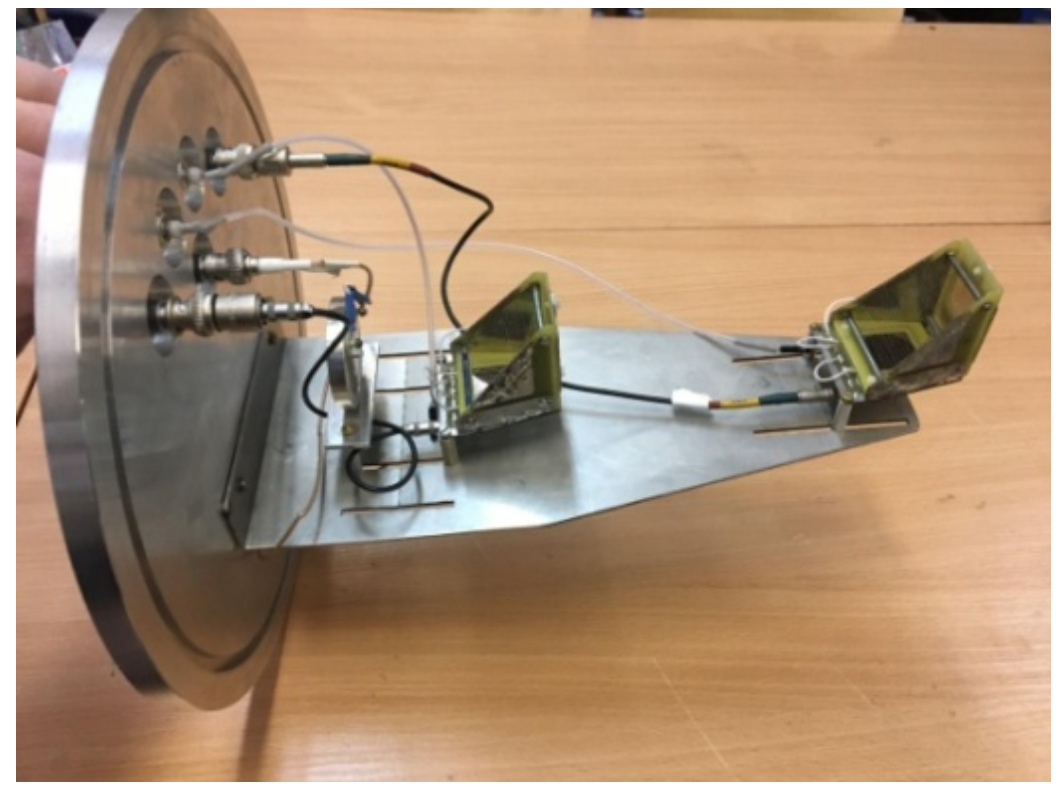

Figure 5. General view of the time-of-flight spectrometer.

The ${ }^{252} \mathrm{Cf}$ source and detectors were located in a vacuum chamber at a pressure of $5 \times 10^{-6}$ torr, in order to eliminate the loss of kinetic energy of the fission fragments due to air ionization. The spectrometer used three detectors - two of them based on MCP, giving time "start" and "stop" signals, and the third is silicon detector for measuring the energy of fission fragments. The path length between the start and stop MCP detectors is $150 \mathrm{~mm}$, and from the start MCP detector to the silicon detector is $200 \mathrm{~mm}$. The diameter of the source is $5 \mathrm{~mm}$. Figure 6 shows a diagram of the decay products yield from their energy and time-of-flight.

In this paper, we obtained the results of comparative measurements of the time-of-flight of spontaneous fission fragments of ${ }^{252} \mathrm{Cf}$ for two methods of measuring the time-of-flight. The results are shown in Figure 7.

As a result, a comparison of the time distributions (Figure $7 b$ ) shows that the time resolution is two times worse when using a Si detector, compared to using two MCP detectors (see Table 1). It should be noted that, despite the worst time-of-flight resolution with a silicon detector, its use is necessary for measuring 


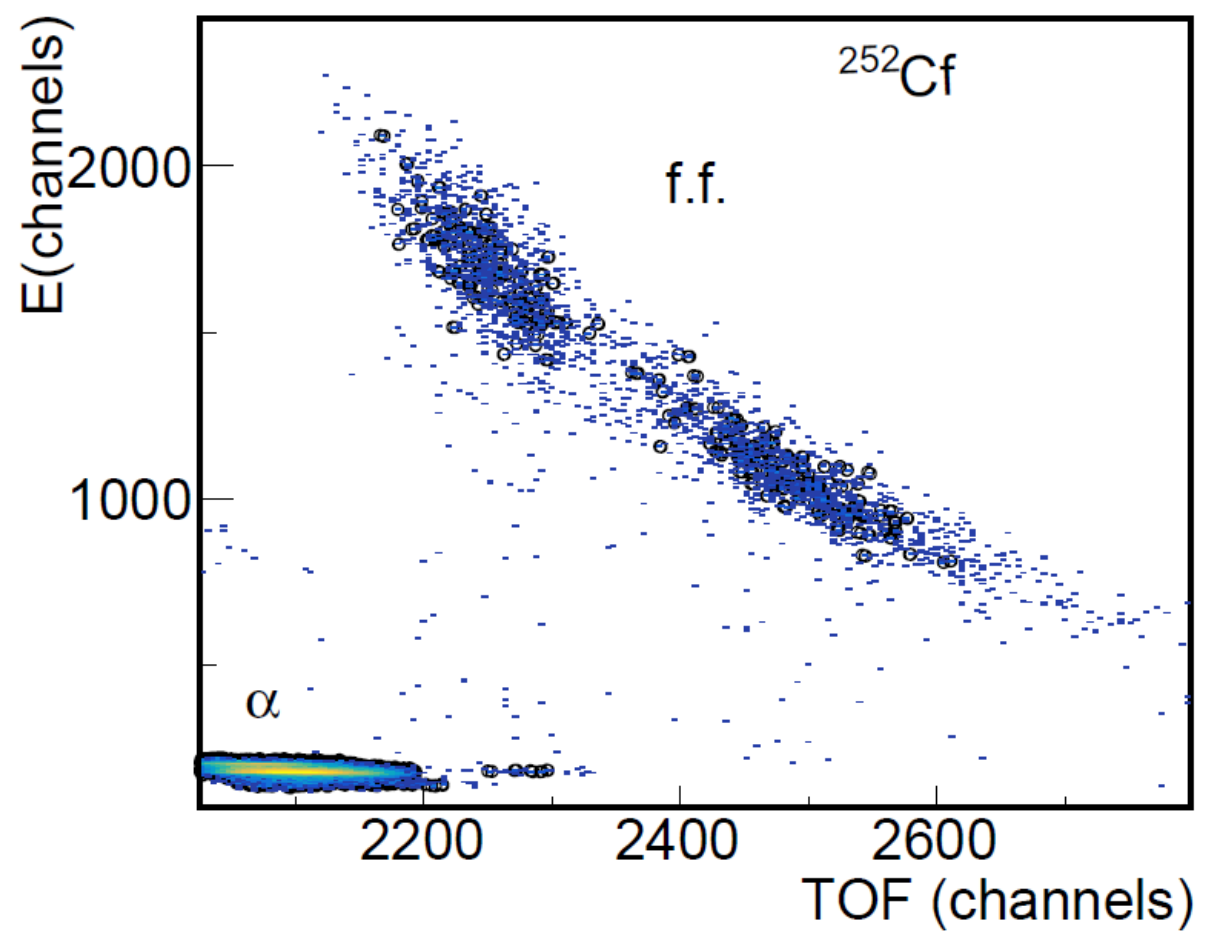

Figure 6. Diagram of the fission products yields of ${ }^{252} \mathrm{Cf}$ from their energy and time-of-flight.
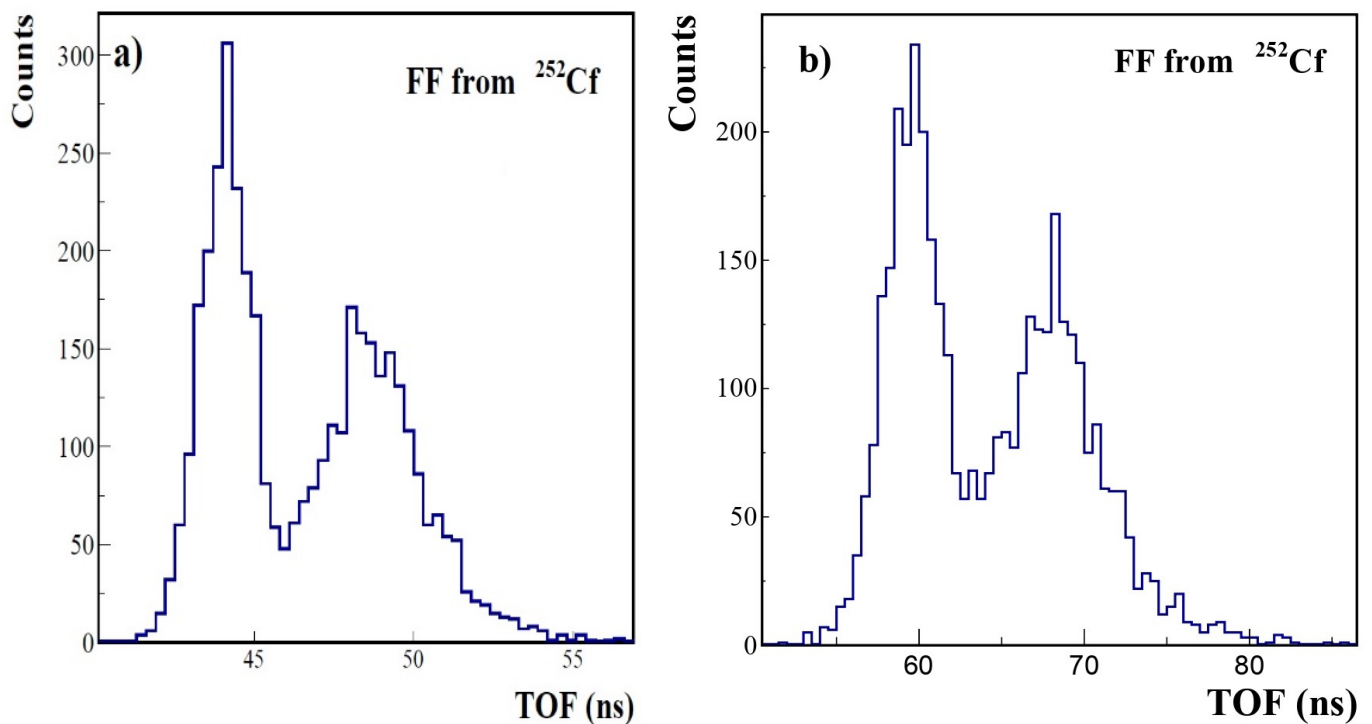

Figure 7. Time-of-flight distribution for ${ }^{252} \mathrm{Cf}$ fission fragments: a) "start" and "stop" signals from the MCP detectors; b) "start" and "stop" signals from the MCP and Si detectors.

the energy of the registered products, which in turn is necessary for calculating the mass of products. The results of such a measurement of induced fission on a beam of ${ }^{48} \mathrm{Ca}$ ions at $310 \mathrm{MeV}$ energy are given below. Mass distributions were obtained from the measured velocities and energies $E_{l}=105.33 \mathrm{MeV}$ and $E_{h}=$ 80.7 MeV of the ${ }^{252} \mathrm{Cf}$ fission fragments using formulas (2 and 3). The following results were obtained: the mass of the light fragment $M_{l}=116 \mathrm{amu}$, and the mass of the heavy fragment $M_{h}=140$ amu. Figure 8 shows the mass distribution of fission fragments of ${ }^{252} \mathrm{Cf}$ in comparison with the results of [19], in which the 
mass values were obtained: the mass of the light fragment $M_{l}=108.6 \mathrm{amu}$, the mass of the heavy fragment $-M_{h}=143.4 \mathrm{amu}$, as well as the energy values: $E_{l}$ $=104.6 \mathrm{MeV}$ and $E_{h}=79.7 \mathrm{MeV}$.

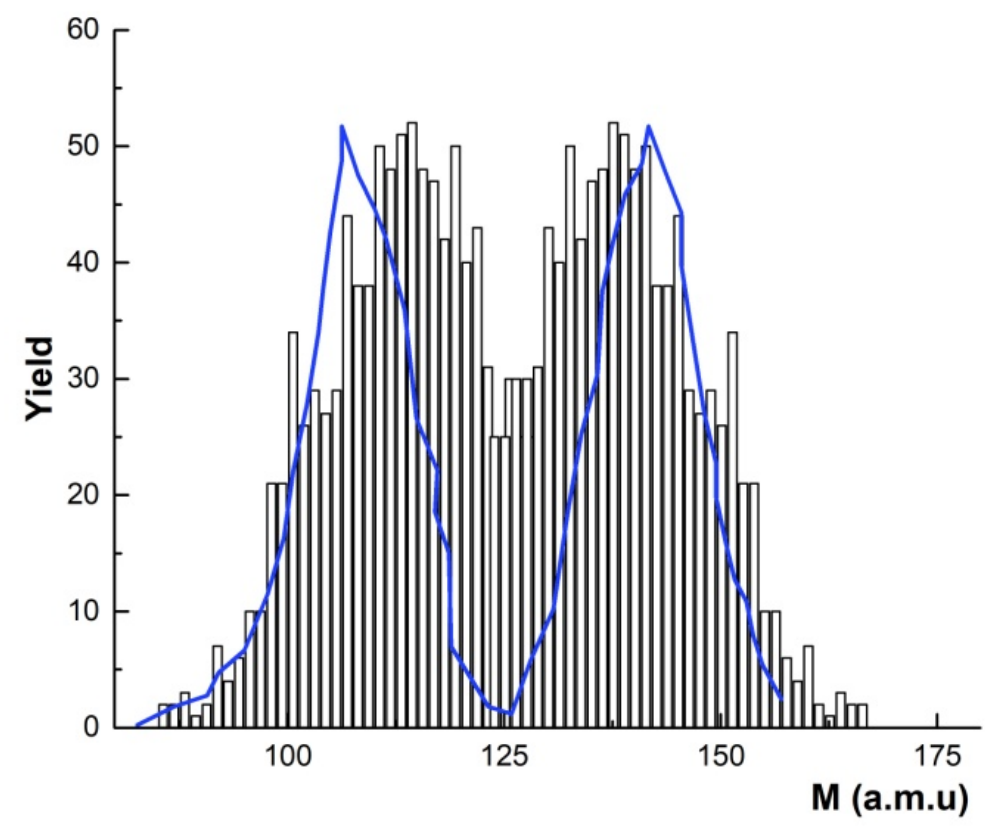

Figure 8. Mass distribution of fission fragments of ${ }^{252} \mathrm{Cf}$ : the histogram on the graph shows the experimental data, the blue line shows the results of the mass distribution from the work [19].

\section{Experimental facility for measuring induced fission fragments in reaction with a ${ }^{48} \mathrm{Ca}$ ion beam}

The experiment was carried out on ${ }^{48} \mathrm{Ca}$ ion beams with energy of $310 \mathrm{MeV}$ at the MAVR facility FLNR JINR. The reaction products were registered by two time-of-flight systems. A schematic picture of the created two-arm time-of-flight spectrometer is shown in Figure 9. The first time-of-flight system includes a MCP detector with a size of $33 \mathrm{~mm}$, a thickness of $0.32 \mathrm{~mm}$, and Si detector with a thickness of $300 \mu \mathrm{m}$ and a size of $55 \times 55 \mathrm{~mm}^{2}$. The distance from the target to the MCP is $200 \mathrm{~mm}$, the path length between the MCP detector and the Si detector is $200 \mathrm{~mm}$. The second system is represented by a high-frequency accelerator generator and a Si detector.

After the interaction of an ion beam, for example, ${ }^{48} \mathrm{Ca}$ with a target, fragmentlike products and elastically scattered ions are registered using time-of-flight arms. Other products that fly out at face angle (for example, alpha particles) are detected by detectors placed in the focal plane of the MAVR magnetic analyzer (MSP-144).

To form the beam profile, the magnetic optics of the ion conductor number 9 were used at cyclotron U-400, which supplemented with a system of diaphragms. The beam profile was monitored using two profilometers. In the experiment a $2 \mathrm{mg} / \mathrm{cm}^{2}$-thick ${ }^{238} \mathrm{U}$ target on a $1.5 \mu \mathrm{m}$-thick titanium substrate were used. 


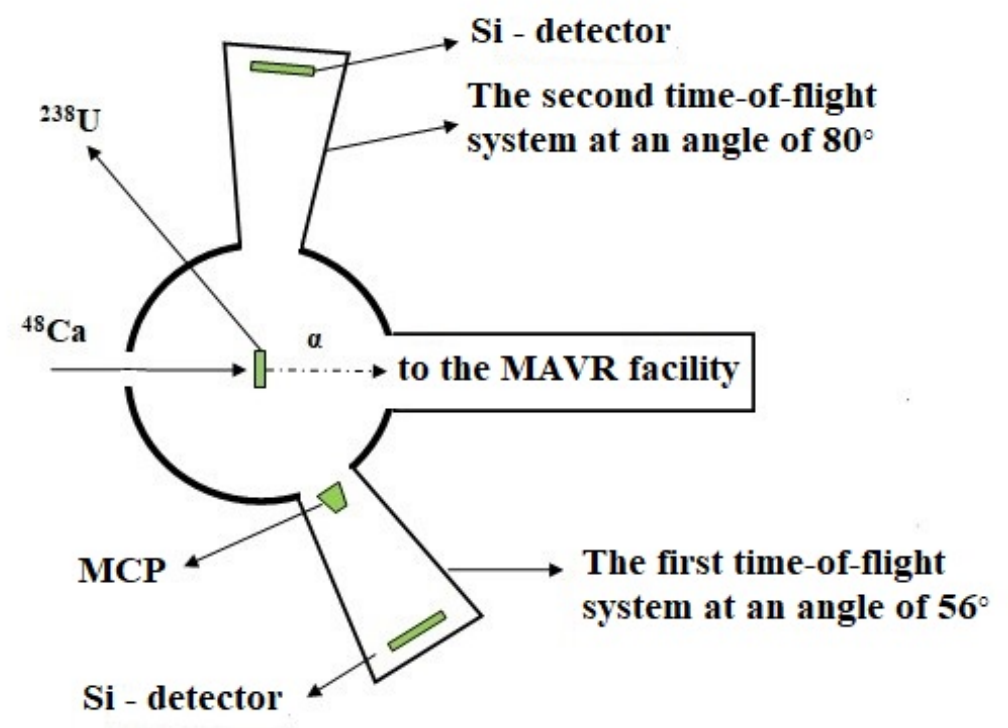

Figure 9. Diagram of a two-arm time-of-flight spectrometer consisting of a reaction chamber and time-of-flight arms based on MCP detectors and Si detectors with an area of $55 \times 55 \mathrm{~mm}^{2}$.

\section{Electronics block scheme}

For measurements by the time-of-flight method, the inverse time method was used, in which the signal from the Si detector at an angle of $56^{\circ}$ is used as a "start", and the pulses from the MCP detector serve as a "stop".

From the Si detector, the starting pulse enters the charge-sensitive preamp PA. From the charge-sensitive preamp, the signals are sent to both the fast (FA) and the spectrometric amplifier (SA), and after the SA, the signal is sent to the ADC. And the signals of the fast amplifier are sent to the fast discriminator CFD, which is intended to time binding. The standard signals from the fast discriminator are sent to the TAC start input. The stop input receives signals from another fast discriminator, which generates signals from the MCP (Figure 10):

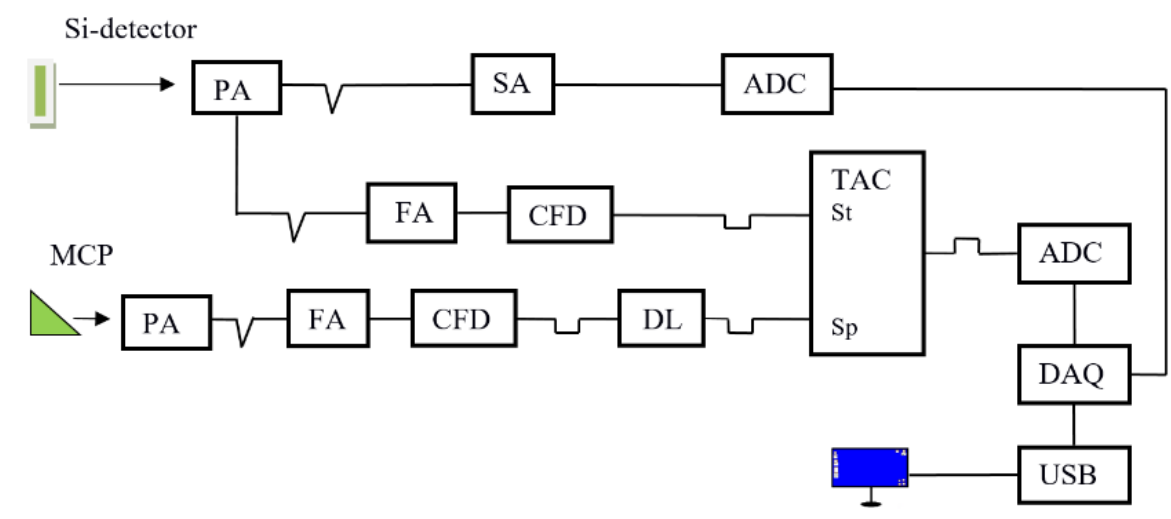

Figure 10. Block scheme of electronics when using a signal from the MCP and Si-detector.

\section{Experimental results}

The "start" signal was taken from the fast output of the discriminator connected to the semiconductor detector in both cases. In addition to obtaining the time 
stamp from our MCP detector, a signal from the high-frequency system of the U-400 accelerator was also used. Figure 11 shows the two-dimensional yields of the reaction products ${ }^{48} \mathrm{Ca}+{ }^{238} \mathrm{U}$ from the time of flight and their energies: a) a time matrix using a binding based on the radiofrequency (RF) system of the accelerator. b) time stamp detector based on microchannel plates:
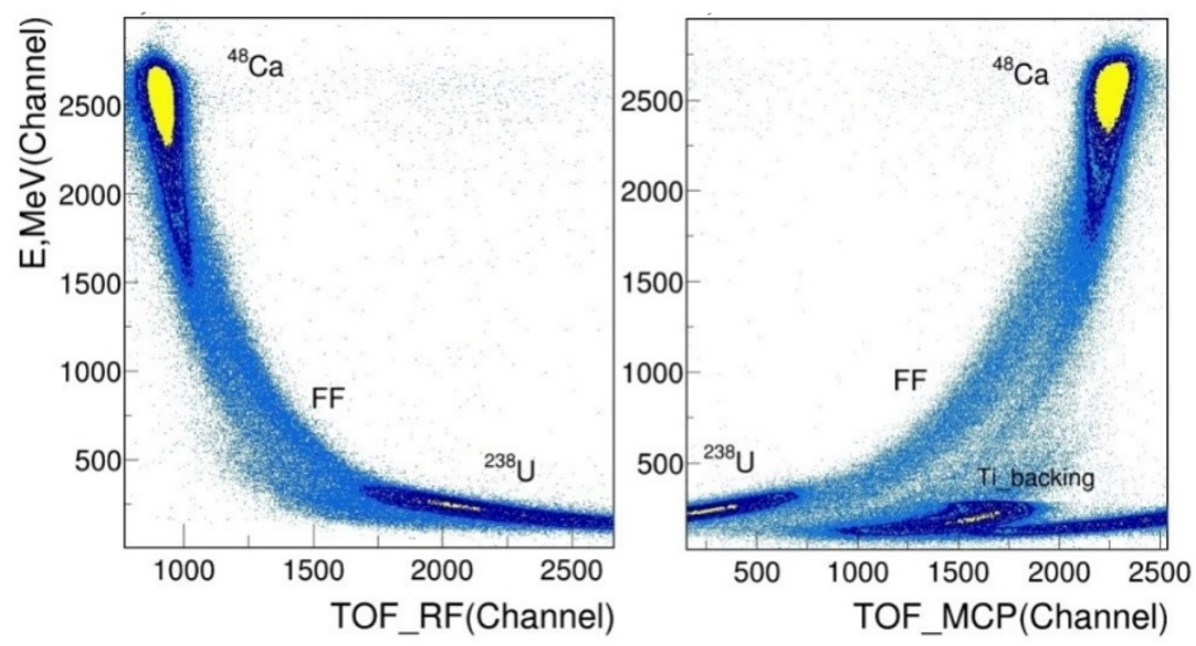

Figure 11. Two-dimensional matrix of the yield of reaction products ${ }^{48} \mathrm{Ca}+{ }^{238} \mathrm{U}$ from the time of flight and their energies: a) Time matrix using a binding based on the RF accelerator system; b) Time stamp detector based on microchannel plates.

As follows from the above (Figure 11b), the time resolution in the case of using a detector based on the MCP is higher compared to the case of obtaining a "time" stamp from the RF system of the accelerator. There is also a qualitative difference in the measured spectra in the ability to more reliably distinguish the region of forced fission fragments from other reaction channels, which is given below.

The values of the registration efficiency of reaction products using this detector are close to $100 \%$ for all product masses. This efficiency is determined by the "transparency" of the grids used. The time resolution was measured from the elastic scattering peak of ${ }^{48} \mathrm{Ca}$ and was $1.5 \mathrm{~ns}$, which is explained by the contribution of the intrinsic energy spread of the ${ }^{48} \mathrm{Ca}$ ion beam (1\%) and the absorption effect and rescattering in the target used. It should also be taken into account that the use of a semiconductor detector as a "start" signal detector leads to deterioration in the intrinsic time resolution to the value of $0.3 \mathrm{~ns}$ due to a longer pulse rise front.

In both cases, a good separation of the elastic scattering products and the fragments of induced fission FF (fission fragments) are seen. However, in the second case (Figure 11b), the best separation of the reaction products by their channels is seen: FF - fission fragments, ${ }^{48} \mathrm{Ca}$ - elastic beam scattering, ${ }^{238} \mathrm{U}-$ elastic uranium scattering. The best time resolution in the case of measurement with a detector based on the MCP makes it possible to clearly distinguish the region of fission fragments, separately from the quasi-elastic and elastic channels.

Based on the measured values of the time of flight and energies, the masses of the registered products were calculated. Figure 12 shows the mass distribution of 
the reaction products ${ }^{48} \mathrm{Ca}+{ }^{238} \mathrm{U}$. The area corresponding to the fission fragments is highlighted quite brightly. The resolution of the elastically scattered ${ }^{48} \mathrm{Ca}$ ions was obtained with an accuracy of FWHM $=5 \mathrm{amu}$ - this low value is explained by the use of a thick target of ${ }^{238} \mathrm{U}\left(2 \mathrm{mg} / \mathrm{cm}^{2}\right)$.

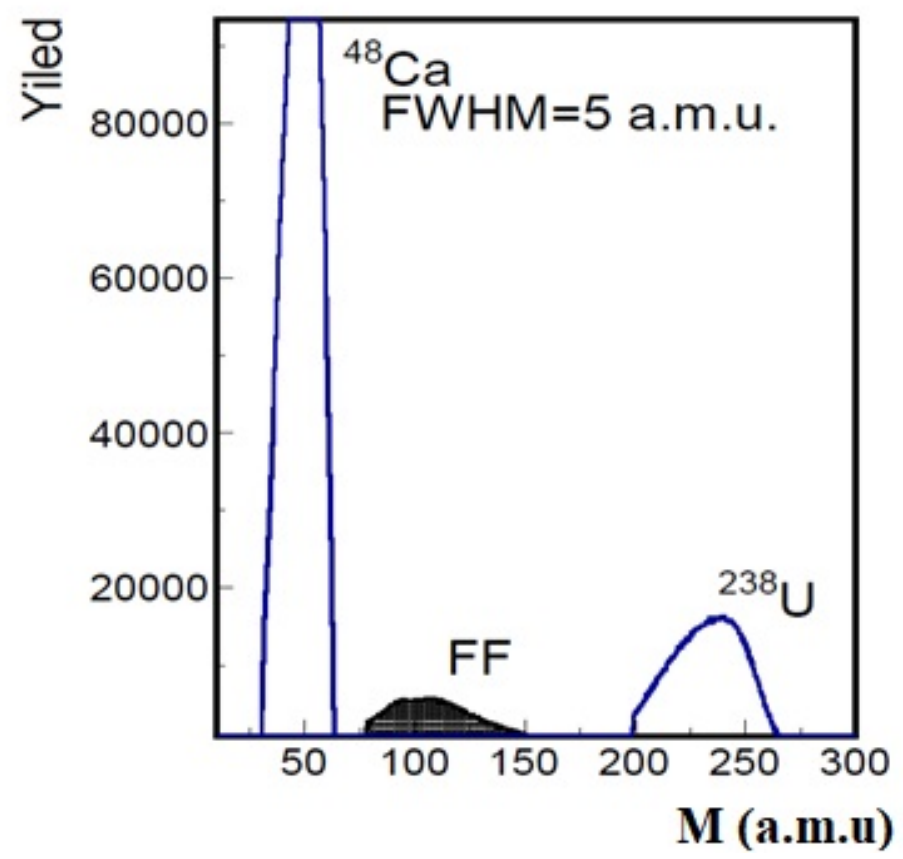

Figure 12. Mass distribution of reaction products ${ }^{48} \mathrm{Ca}+{ }^{238} \mathrm{U}$ : elastically scattered ions ${ }^{48} \mathrm{Ca}$ and ${ }^{238} \mathrm{U}$, FF - fission fragments are well separated from quasi-elastic products.

In addition to measuring the time resolution in various "start-stop" configurations, the efficiency of the detector system used was also measured. According to the measurements, the obtained values of the time resolution and registration efficiency are presented in Table 1.

Table 1. The obtained values of the time resolution and registration efficiency.

\begin{tabular}{c|c|c|c|c}
\hline \multirow{2}{*}{${ }^{226} \mathrm{Ra}$} & Start & Stop & Time resolution, $\mathrm{ns}$ & Efficiency \% \\
\cline { 2 - 5 } & MCP detector & MCP detector & 0.470 & \multirow{2}{*}{60} \\
\cline { 2 - 5 } & MCP detector & Si detector & 0.7 & \multirow{2}{*}{95} \\
\hline \multirow{2}{*}{${ }^{252} \mathrm{Cf}$} & MCP detector & MCP detector & 0.470 & \multirow{2}{*}{100} \\
\cline { 2 - 5 } & MCP detector & Si detector & 0.7 & \\
\hline \multirow{2}{*}{${ }^{48}$ Ca beam } & Si detector & MCP detector & 1.5 & \\
\cline { 2 - 5 } & Si detector & RF accelerator & 2.5 & \\
\hline
\end{tabular}

It follows from the table that the best time resolution is obtained in systems using detectors based on MCP, but with less efficiency when registering alpha particles. The main reason lies in the amplitude distribution of the signals from the anode of the MCP detectors [16]. As shown in [16], alpha particles with energy of about $5 \mathrm{MeV}$ generate to an average of 2-3 secondary emission electrons, and more than 100 electrons per fission fragment. 


\section{Conclusion}

In this paper, we demonstrated a method for studying nuclear fission based on measuring the time of flight of fission fragments. Two methods of measuring the time of flight were used: the "stop" signal was generated using the RF system of the accelerator and using a specially designed time stamp detector based on microchannel plates. The paper shows the advantage of measuring the time of flight by the second method, i.e. using a time stamp detector based on microchannel plates.

The created system makes it possible to register elastically scattered ions, so it is used as a monitoring system for the quality and composition of the beam on the target of the MAVR facility.

The time resolution was measured from the elastic scattering peak of ${ }^{48} \mathrm{Ca}$ and was $1.5 \mathrm{~ns}$, which is explained by the contribution of the intrinsic energy spread of the ${ }^{48} \mathrm{Ca}$ ion beam (1\%) and the effect of absorption and rescattering in the target used.

\section{Acknowledgments}

The authors express their gratitude to Professor Yu.E. Penionzhkevich for his valuable advice in writing the work, as well as to all colleagues for organizing and making a huge contribution to this research. The authors are grateful to B.A. Vorobyov for his help in creating a design for installing detectors necessary for conducting these experiments, as well as to N.V. Aksenov for making targets.

\section{References}

[1] Yu.E. Penionzhkevich et al., Journal of Physics: Conference Series 1555 (2020) 012031.

[2] A.K. Azhibekov et al., Physics of Atomic Nuclei 83 (2020) 93-100.

[3] S. Lukyanov et al., Eurasian journal of physics and functional materials 4 (2020) 274-280.

[4] Yu.Ts. Oganessian, G.G. Gulbekyan, B.N. Gikal, I.V. Kalagin, Proceeding of APAC 2004 International Conference, Gyeongju, Korea (2004) 52-54.

[5] G.G. Gulbekyan et al., Exotic Nuclei Exon 2004 (2005) 643-650.

[6] R. Kalpakchieva, Yu.E. Penionzhkevich, H.G. Bohlen, Physics of Elementary Particles and Atomic Nuclei 29 (1998) 832-885.

[7] Yu.E. Penionzhkevich, Soros Educational Journal 1 (1995) 92-96.

[8] K.A. Kuterbekov et al., Chinese Journal of Physics 55 (2017) 2523-2539.

[9] A.M. Kabyshev et al., Journal of Physics G: Nuclear and Particle Physics 45 (2018) 025103.

[10] Yu.E. Penionzhkevich, Eurasian Journal of Physics and Functional Materials 2 (2017) 19-39.

[11] Yu.E. Penionzhkevich, R.G. Kalpakchieva, Light nuclei near the boundary of neutron stability (JINR, Dubna, 2016) 383 p. 
[12] B.V. Rybakov, V.A. Sidorov, Prompt neutron spectrometry (Atompublisher, Moscow, 1958) $176 \mathrm{p}$.

[13] N.I. Venikov, N.I. Chumakov, Atomic Energy 17 (1964) 503-504.

[14] Yu.P. Gagrsky, B.N. Markov, V.P. Perelygin, Registration and spectrometry of fission fragments (Energoatompublisher, Moscow, 1992) 312 p.

[15] G.M. Ter-Akopian et al., Physics of Elementary Particles and Atomic Nuclei 28 (1997) 1357-1388.

[16] V.D. Dmitriev, S.M. Lukyanov, Yu.E. Penionzhkevich, D.K. Sattarov, IET Electric Power Applications (1982) 7-18.

[17] D. Aznabaev et al., Physics of Elementary Particles and Atomic Nuclei, Letters 16 (2019) 620-626.

[18] E.M. Kozulin et al., Instruments and Experimental Techniques 51 (2008) 44-58.

[19] P. David et al., Physics Letters B 60 (1976) 445-447. 